
Revue canadienne de chimie

\title{
Peptide dendrimers: drug/gene delivery and other approaches
}

\begin{tabular}{|c|c|}
\hline Journal: & Canadian Journal of Chemistry \\
\hline Manuscript ID & cjc-2017-0242.R1 \\
\hline Manuscript Type: & Mini Review \\
\hline Date Submitted by the Author: & 14-Jun-2017 \\
\hline Complete List of Authors: & $\begin{array}{l}\text { Santos, Soraya; University of Sao Paulo, Pharmacy } \\
\text { Gonzaga, Rodrigo; Universidade de Sao Paulo Faculdade de Ciencias } \\
\text { Farmaceuticas, Pharmacy } \\
\text { Silva, João Vitor; Universidade de Sao Paulo Faculdade de Ciencias } \\
\text { Farmaceuticas, Pharmacy } \\
\text { Savino, Débora; Universidade de Sao Paulo Faculdade de Ciencias } \\
\text { Farmaceuticas, Pharmacy } \\
\text { Prieto, Diego; Universidade de Sao Paulo Faculdade de Ciencias } \\
\text { Farmaceuticas, Pharmacy } \\
\text { Shikay, Jennifer; Universidade de Sao Paulo Faculdade de Ciencias } \\
\text { Farmaceuticas, Pharmacy } \\
\text { Silva, Renan; Universidade de Sao Paulo Faculdade de Ciencias } \\
\text { Farmaceuticas, Pharmacy } \\
\text { Paulo, Lucas Henrique; Universidade de Sao Paulo Faculdade de Ciencias } \\
\text { Farmaceuticas, Pharmacy } \\
\text { Ferreira, Elizabeth; Universidade de Sao Paulo Faculdade de Ciencias } \\
\text { Farmaceuticas, Pharmacy } \\
\text { Vargas, Jeanine; Universidade de Sao Paulo Faculdade de Ciencias } \\
\text { Farmaceuticas, Pharmacy }\end{array}$ \\
\hline $\begin{array}{r}\text { Is the invited manuscript for } \\
\text { consideration in a Special } \\
\text { Issue?: }\end{array}$ & Dendimers \\
\hline Keyword: & Dendrimer, Peptide dendimer, Drug delivery, Gene delivery \\
\hline
\end{tabular}

\section{SCHOLARONE ${ }^{m}$ \\ Manuscripts}




\title{
PEPTIDE DENDRIMERS: DRUG/GENE DELIVERY AND OTHER APPROACHES
}

\section{SANTOS, S.S., GONZAGA, R.V., SILVA, J.V., SAVINO, D.F., PRIETO, D., SHIKAY, J.M., SILVA, R.S.; PAULO, L.H.A.; FERREIRA, E.I., GIAROLLA, J*.}

*Corresponding author: jeanineg@usp.br

Faculty of Pharmaceutical Sciences, University of Sao Paulo, Brazil

\begin{abstract}
Dendrimers are versatile hyperbranched molecules, which have deserved attention, especially for their potential in many applications, including biological. Peptide dendrimers comprises interesting classes of dendrimers and their use has been emphasized as drug/bioactive compound delivery system, mostly in the antineoplastic area. The bioactive molecules can be covalently linked or entrapped inside the peptide derivative. Self-assembled nanocarriers are a recent trend in the design of potential delivery systems and $\mathrm{pH}$-sensitive carriers, one of their methods, have been designed to control their systems. In addition, the use of targeting peptides or other specific groups that direct the drug/bioactive compounds to specific organs is an important trend in the search for better drug delivery systems. Recent examples have been given in the literature showing that gene delivery as other important peptide dendrimers applications. It is worth emphazing that some peptide dendrimers show activity per se, without bioactive compounds. Immune compounds and vaccines were shortly presented herein, as well as uses of other peptide dendrimers briefly discussed in this review, which encompasses around 10 years of work.
\end{abstract}

Keyword: dendrimer, peptide dendrimer, drug delivery, gene delivery.

\section{INTRODUCTION}

Peptide dendrimers are hyperbranched macromolecules built by covalent bonds, in which dendrons, core or surface functional groups are composed of peptides. Their molecular weight ranges from $2 \mathrm{kDa}$, small structures, to $100 \mathrm{kDa}$, large protein-like structures, arranged in 2 to 32 generations. Two features contribute to the complexity of peptide dendrimers: generation number and surface terminal groups. These molecules 
provide a great chemical diversity and different applications, especially in biotechnological sciences. Some of them include drug and gene delivery, biomedical diagnosis, vaccines for multiple diseases, protein mimetics, and enzyme catalysts. ${ }^{1-5}$ The diversity of applications are due, mainly, to better stability of the structures and to their similarity to biomacromolecules. ${ }^{6}$

Generally, peptide dendrimers are classified according to the bond between the peptide and the dendron as covalent and non-covalent structures and can be synthesized as follows: convergent (most common) and divergent method. Their synthesis is usually carried out through solid phase ${ }^{7}$, by means of adequate resins, employing specific protecting groups.

Interesting reviews have been published reporting general synthetic methods employed to obtain peptide and glycopeptide dendrimers, such as solid-phase synthesis to chemoselective and orthogonal binding. ${ }^{1,2,8,9}$. Jan Jezek's group also reported interesting and relevant papers related to peptide and glycopeptide dendrimers. ${ }^{10,11,12,13}$

The combination of dendrimers and bioactive peptides properties through the letter may provide synergic action due to: (1) polyvalence, which can raise biological effects of the conjugated-peptides; (2) interaction with many receptors at the same time; (3) protein-like characteristics, mimicking biological structures, for instance artificial proteins. Moreover, they allow endowing biocompatibility and biodegradability to the final structure. ${ }^{9}$

There are mainly three kinds of peptide dendrimers:

The first has its branches grafted or decorated with unnatural amino acids or organic groups and its core and surface groups may be decorated with peptide/proteins. They present high generations, usually being the largest compounds. In a review by Wan and Alewwod ${ }^{3}$ (2016), the synthesis and biological applications of those dendrimers was highlighted. Examples given involve dendrimers with a polymeric core (as Poly(amidoamine) - PAMAM - dendrimer) and multiple peptide unities covalently coupled as surface groups.

The second type, generally, the smallest molecules, comprises branches composed of polyamino acids. Since 1980 s, when the research with peptide dendrimers started, poly-lysine dendrimer has been one of the most widely used. ${ }^{3}$

The third type, developed by $\operatorname{Tam}^{8}$ (1988), behaves as multiple Antigen Peptide (MAP), and it is constituted of amino acids in the core and peptide chains as the surface 
groups. They have been employed in biological and biochemical fields, especially as immunogens. ${ }^{1,8}$

Based on the previous characteristics and considering the importance of peptide dendrimers, the main objective of this review was to explore their different biomedical applications in the last 10 years. In this context, the design of new drug delivery systems, gene delivery, and their activity as therapeutic agent, will be emphasized. In addition, other biological uses will be presented and briefly discussed herein.

\section{Drug delivery}

Drug delivery has been a challenge towards improving bioavailability and, specially, increasing selectivity, which lowers toxicity. ${ }^{14}$

Drugs can be bound to the dendrimers, either leading to prodrugs or targeted $\operatorname{drugs}^{15}$ or be encapsulated in these nanostructures, through weak interactions. ${ }^{16}$ It is worth noting that several targeting strategies based on the fact that carbohydrates have the ability to target active sites, have been explored. Mannose, for example, can be recognized by macrophages and also neutralize cationic charges on dendrimer surface, which increases toxicity ${ }^{17}$ Other cells, as hepatocytes, show receptors sensitive to galactose interaction, allowing liver selectivity for some drugs. ${ }^{18}$

Encapsulated-dendrimers have attracted interest in recent years due to their unique properties, such as well-defined size, globular shape, and internal empty space, allowing encapsulate drugs through electrostatic, complex formation, van der Waals forces and H-bonding interactions. Their properties permit increasing drug/bioactive compound water solubility as well. ${ }^{19}$ Poly(amidoamine) (PAMAM) has been employed to encapsulate poorly soluble bioactive compounds, increasing solubility successfully, enhancing their activity. ${ }^{20}$

Many approaches have been used to achieve drug delivery. Self-assembled nanocarrier is a recent trend in its potential design. There are interesting examples in the literature concerning this mechanism of drug delivery. ${ }^{6,21}$ Verma and coworkers ${ }^{22}$ (2015) reported urea and triazole as core in the planning of self-assembling peptide dendrimers. The branches are composed of glutamic and aspartic acids. The branches coupling through $\mathrm{N}$-terminals by a carbonyl group provides the urea core formation, which has benefits, such as strong intrinsic self-assembly and anionic binding properties. In this context, urea function is an interesting entity, which may be employed as core for the development of self-assembling dendrimers. 
Transdermal delivery has also attracted interest in the academic community and there are some peptide nanocarriers designed for this application. Conjugation with iontophoresis was described by Mutaliki and colleagues ${ }^{23}$ (2013) (Figure 1), who synthesized peptide dendrimers, constituted of arginine and histidine, as terminal groups and composed of 4, 6 and 8 molecules of each amino acids. Their stability was tested in receptor solution on epidermis, dermis and skin, and showed that there was not noticeable degradation, with $98.9 \%$ stability in all these tests after $6 \mathrm{~h}$ at room temperature. In passive diffusion, dendrimers with higher molecular weight did not permeate across the skin. On the other hand, dendrimers with lower molecular weight permeated across the skin, with good stability profile. Iontophoresis increased significantly the permeation of all compounds, meaning that permeation is dependent on molecular weight rather than on the positive charges of the amino acids.

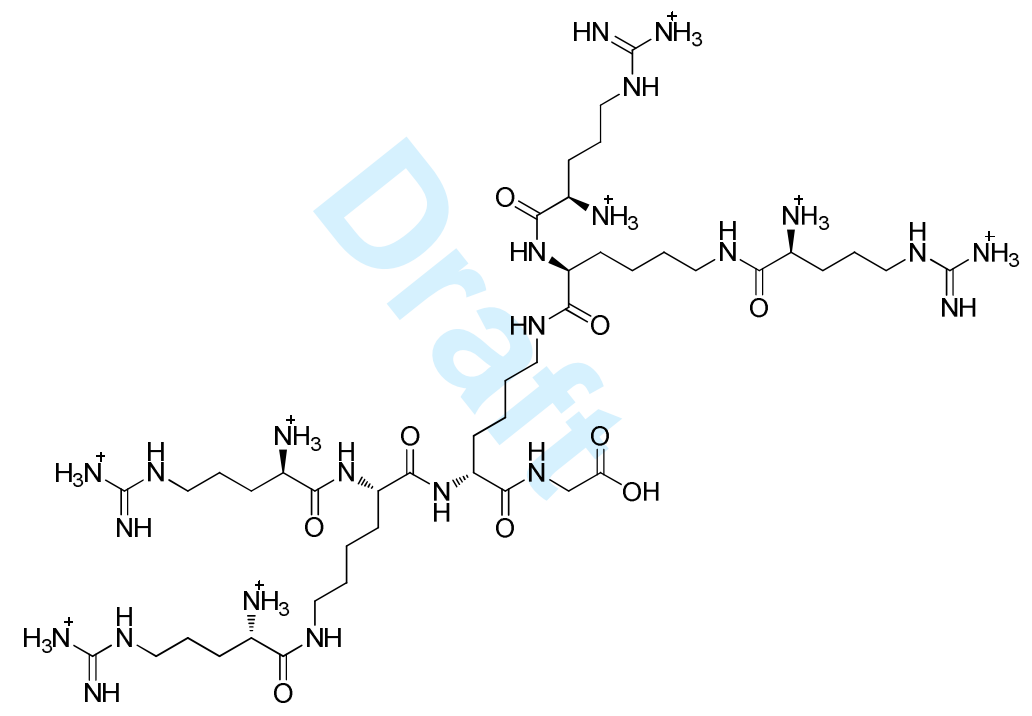

Figure 1 - Nanocarrier designed for transdermal delivery.

The capacity of peptide dendrimers to cell-internalize has been a challenge to the delivery of some drugs, mainly in oncology. With the purpose of finding chemical architectures of peptide dendrimers, Yan and coworkers ${ }^{24}$ (2015) conjugated CellPenetrating Linear Peptides, CPPs, with PAMAM dendrimer. The conjugates were labeled with a fluorescent dye, boron-dipyrromethene, BODIPY (BP), and linked to Tat-peptide (GRKKRRQRRRPQ), which is derived from the transactivator of human immunodeficiency transcription (BPT). The internalization showed to be dependent on the amount of Tat-peptide conjugated with PAMAM. Submitted to cytotoxicity tests, PAMAM conjugate was less toxic than free dendrimer. This effect could be related to 
positive charge and to the concentration of Tat-peptide in the conjugate. Using tumor cells to evaluate the internalization, the authors observed the rate of BPT in these cells was 3-fold than that of BP, using the same doses. Despite being detected in other tissues, the highest amount of the conjugate was found in the tumor tissue. Moreover, $200 \mathrm{~nm}$-particles demonstrated greater uptake than $5.5 \mathrm{~nm}$-particles, as the latter are rapidly excreted by the kidneys.

Selective deliver to the brain is very important for some Central Nervous System (CNS) drugs or even to antitumor agents. Trying to find a suitable peptide dendrimer structure to assist this kind of drug delivery, Jiang and coworkers ${ }^{25}$ (2016) developed a peptide dendrimer based on PAMAM PEGylated. Previous studies showed that PEGylation of PAMAM promoted longer blood circulation and less toxicity. PAMAM dendrimers were modified with PEG chains and Pep-1, a linear small peptide that is a specific ligand of interleukin-13 alfa-2 receptor, overexpressed in glioma cell lines. Both Pep-PEG-PAMAM and PEG-PAMAM showed that uptake depends on the concentration. No toxic effects were observed in cell lines, which kept viable after the assay. Further tests showed that Pep-PEG-PAMAM have had higher accumulation and 2-fold higher fluorescence intensity in tumor than PEG-PAMAM.

Although many therapeutic classes of drugs face delivery problems, most examples in literature are related to chemotherapeutic agents, especially to anticancer drugs. ${ }^{26}$ The reason for increasing interest in this class of drugs and bioactive agents is, usually due to their high toxicity and low selectivity, which frequently leads to interruption of the treatment or death. Thus, proper and specific drug delivery systems would be of utmost importance and peptide dendrimers are able to achieve that purpose.

Examples of anticancer drug delivery systems and other drugs are as follows.

\section{Anticancer drug delivery systems}

New approaches to reduce toxicity and improve bioavailability are underway for cancer therapy like dendrimer nanoparticles used as a vehicle for drug delivery. ${ }^{27}$

Based on the difference between microenvironment of the tumor and of the normal tissues, targeting moieties to specific cells have been explored. Aiming at selectivity, sugar moieties, folic acid, antibody, peptide and epidermal growth factor have been used to concentrate drugs/bioactive compounds in neoplastic cells through diverse mechanisms. 
Receptor-mediated endocytosis (RME) or the effect of enhanced permeability and retention (EPR) enzyme-responsive and $\mathrm{pH}$-responsive strategies have also been applied towards toxicity decrease and selectivity enhanciment. ${ }^{28,29}$

In 2015, Oledzka and colleagues ${ }^{30}$ designed macromolecular drug delivery systems containing poly-lysine dendrimers, surface groups composed of arginine-6oligomer and amphiphilic copolymer poly(3-hydroxybutyrate (PHB)-poly(ethylene glycol) (PEG)-PHB, as core. In this context, they proposed a biodegradable poly-(Llysine) dendrimer presenting arginine as functionalized-end aiming at synthesizing a chemically polyvalent copolymer. This might be conjugated to drugs through covalent bonds and be further. Colchicine, which has not been used as a chemotherapeutic agent due to its cytotoxicity, ${ }^{31}$ was one of the drugs tested.

Peptide dendrimers comprising amino acid combinations, as aspartate, histidine and serine, were conjugated to carbohydrates in their surface with antineoplastic drugs as delivery systems. Colchicine was conjugated to dendrimers and once it was coupled with glycopeptide dendrimers, it presented decrease of toxicity and increase of cellular uptake, when compared to free colchicine. Probably, the proteolysis is the mechanism responsible for the uptake and the intracellular drug release in cancer cells. ${ }^{9}$

Despite doxorubicin (DOX) being one of the most potent anticancer drug, its application is limited by toxicity, such as cardiotoxicity, which causes cardiomyopathies leading, in some circumstances, to death. ${ }^{28,32}$ This has been a great challenge to the use of this drug in cancer chemotherapy and, therefore, many dendrimer peptides have been strategically used as an alternative to overcome its high toxicity and low selectivity as well.

Heparin is a natural water-soluble polysaccharide, which belongs to glycosaminoglycan family and has been used as anticoagulant drug. In addition, it can inhibit tumor growth and metastasis through recognition among selectins, heparanases, and growth factors. Heparin was dendronized with poly(L-lysine) and conjugated by $\mathrm{pH}$-sensitive bonds with DOX (dendronized heparin-DOX conjugate nanoparticles selfassembled) (Figure 2) for the treatment of breast cancer. Histological analysis after using drug-dendron conjugate did not indicate toxicity in comparison with free DOX, demonstrating high antitumor activity on breast cancer cell line (4T1), antiangiogenesis effects and induced apoptosis. According to the authors, the dendronized heparin-DOX conjugate may be a background for the design of biosafe nanoparticles and efficient drug delivery systems. ${ }^{33}$ 


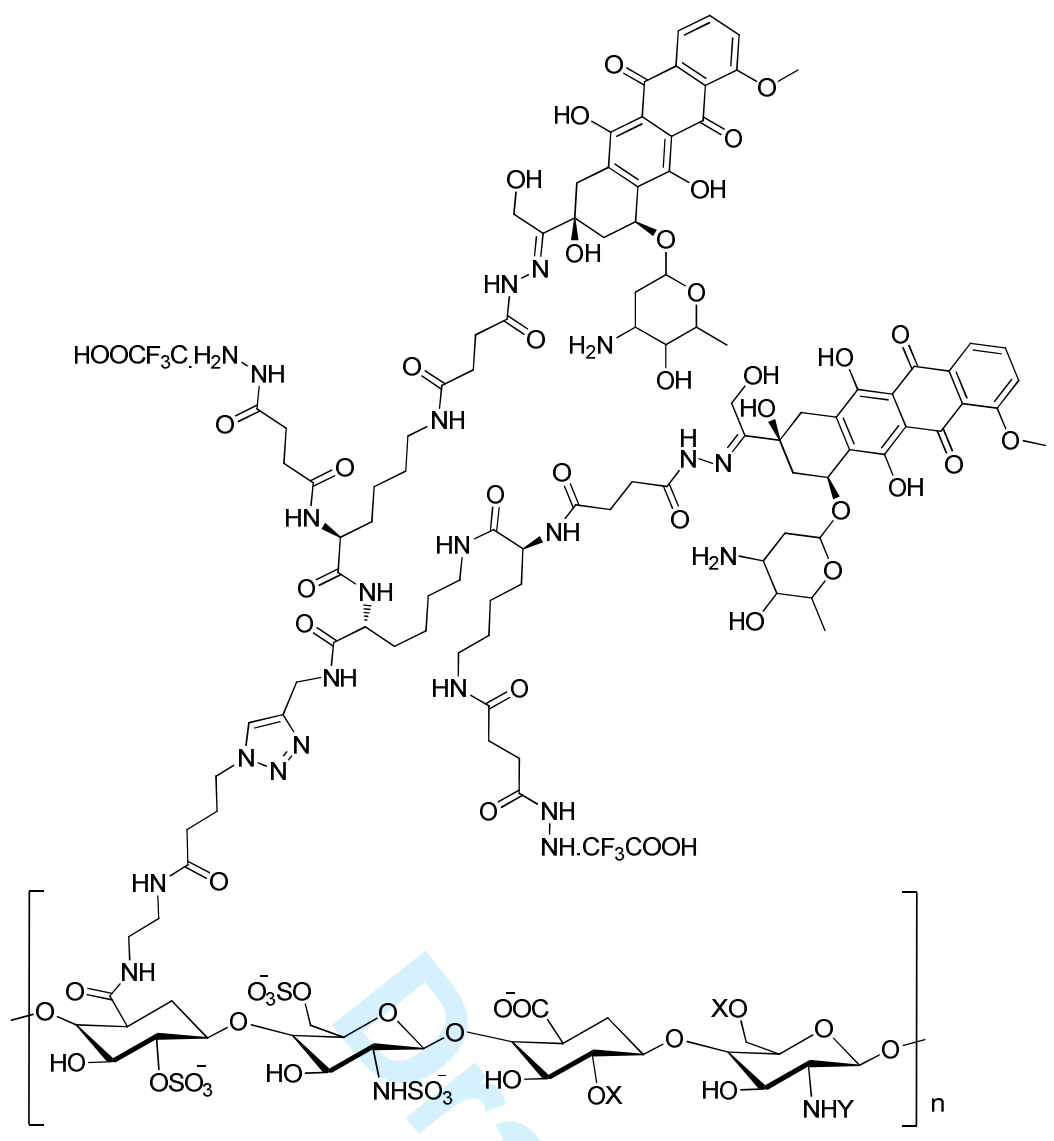

Figure 2 - Heparin dendronized with poly(L-lysine) and conjugated by $\mathrm{pH}$-sensitive bonds with DOX (dendronized heparin-DOX conjugate nanoparticles self-assembled).

The use of amphiphilic dendrimers able to self-assemble is also a new approach directed to selectivity in general and of DOX in particular. ${ }^{3,34}$ mPEGylated peptide dendrimer-DOX has been designed to be an enzyme-responsive drug delivery vehicle. This drug was attached to the dendrimer surface through an enzyme-responsive tetrapeptide Gly-Phe-Leu-Gly (GFLG), cathepsin B substrate (overexpressed in tumor cells) as directing group, and a spacer. PEG was used to increase blood residency time and tumor accumulation. These derivatives (Figure 3) were able to self-assemble into nanoparticles. Furthermore, in immune-histochemical and histological analysis, they showed high antitumor activity and induced apoptosis on breast tumor model, demonstrating better results than free DOX. According to the authors, mPEGylated peptide dendrimer-DOX conjugate-based nanoparticles may be a promising drug delivery system for breast cancer chemotherapy. ${ }^{34}$ Li and colleagues ${ }^{34}$ (2014) published a similar work using the same approach, the PEGylated dendron connected to GFLG coupled to DOX, generating the enzyme-sensitive amphiphilic conjugate self-assemble 
into nanoparticles negatively charged. This delivery system could be cleaved in the lysosome after endocytosis, releasing the drug for cancer therapy. This drug conjugated demonstrated better antitumor activity in the assays in breast tumor cells than free DOX. In addition, the results of in vivo assays showed increased antitumor activity and biosafety compared to the free drug. These results pointed out the potential of peptides for designing drug delivery systems.
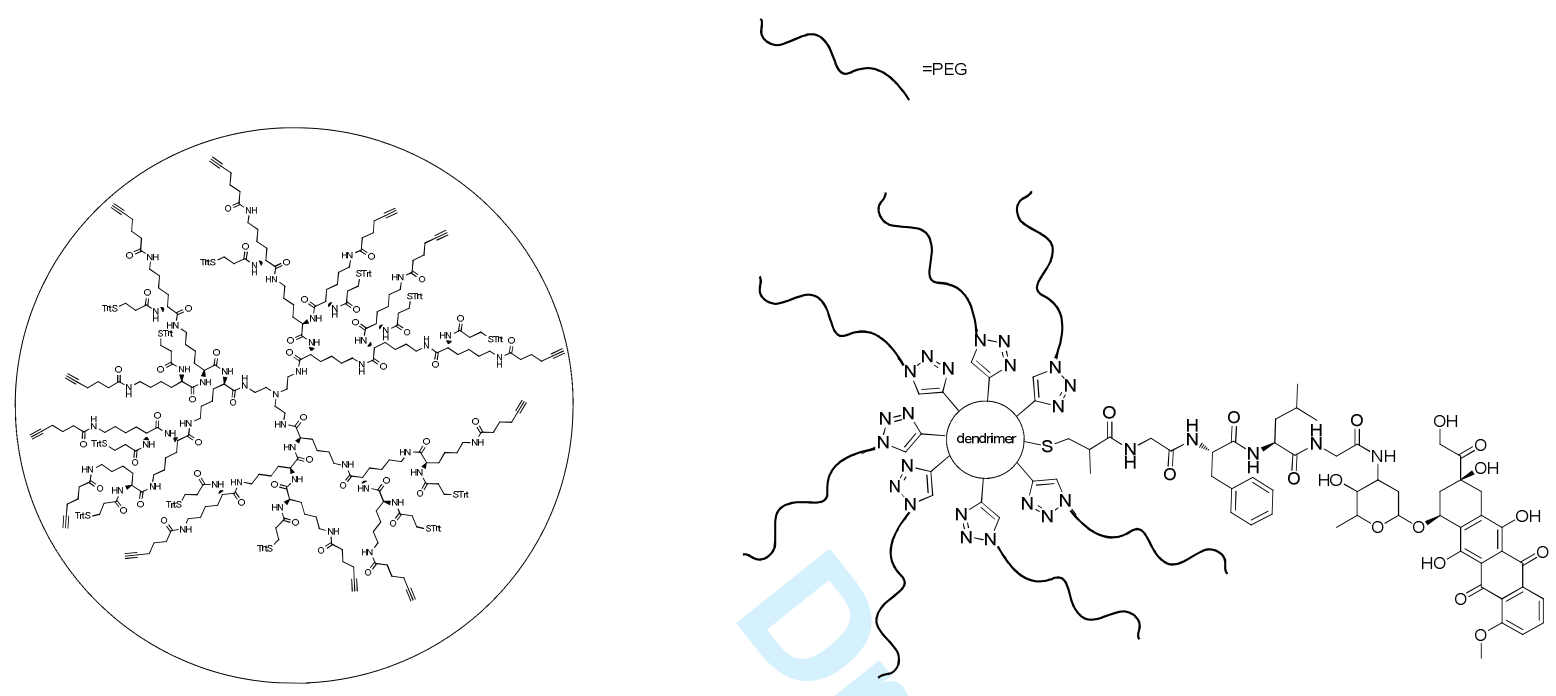

Figure 3 - mPEGylated peptide dendrimer-DOX as enzyme-responsive delivery system.

Cathepsin B-enzyme-responsive tetra-peptide GFLG continues to be extensively explored. Recently, Lee and coworkers ${ }^{35}$ (2015) synthesized peptide dendrimer for DOX delivery. They found to be auto-assembly nanoparticles, containing GFLG as linker, PEG, and drug conjugated to terminal groups of the dendrimer. In vitro experiments were performed using CT26 tumor xenograft for the drug release analysis sensitive to cathepsin-B by fluorescent methods. Peptide dendrimers showed strong fluorescent intensity at the site of the tumor other than the free drug, which was distributed to other areas of the body such as brain, kidneys and liver. Therefore, this system was confirmed as a successful way for targeting cancer cells.

The success of carrier enzyme-sensitive stimulated the research of new enzymatic substrates for antitumor drugs delivery. Recently, Li and colleagues ${ }^{36}$ (2016) synthetized new amphiphilic dendrimer peptide exploring another peptide as enzymesensitive linker. Proline-valine-glycine-leucine-isoleucine-glycine(Pro-Val-GlyLeu-Ile-Gly, PVGLIG), oligopeptide substrate metalloprotease-2 (MMP-2/9)-sensitive was used. Metalloproteases, enzymes overexpressed in many tumor cells, belong to a 
family of proteases that degrade extracellular matrix components. They could be potential candidates to smart drug delivery systems (SDDS), ${ }^{37,38}$ meaning a system in which the drugs are only released in the target tissues/organs at the sites of action, with the adequate rates. The antitumor activity of DOX-conjugate was similar to that of free DOX. Notwithstanding, the in vivo toxicity tests showed that mPEGylated dendronPVGLIG-DOX conjugate was less toxic than the free drug. The results obtained with peptide dendrimer polymer MMP-2/9-responsive demonstrated it is a potential safe and effective drug delivery system for cancer treatment. ${ }^{36}$

Looking for different approaches, there has been an increasing interest in inorganics core like polyhedral oligomeric silsesquioxane (POSS) for dendrimers development. Due to physical and chemical properties, they are three-dimensional structures with eight corners chemically conjugated and increased exponentially peripheral groups, which can make the synthetic processes viable. ${ }^{28}$ In this context, a novel poly(L-glutamic acid) dendrimer with octa(3-aminopropyl) silsesquioxane (OAS) core conjugated with DOX in their peripheral groups was designed. This was performed through $\mathrm{pH}$-sensitive hydrazone bonds and biotin as a specific tumor cells targeting moiety (Figure 4). ${ }^{28}$ This drug-conjugate showed antitumor effects with dual targeting mechanisms, namely, $\mathrm{pH}$-sensitive drug release and cellular internalization of the conjugates through biotin-specific receptors (BSR). The rapid growth of cancer cells requires a greater amount of biotin, which is a micronutrient essential for cell development. Since BSR are overexpressed on cancer cells, biotin could, therefore, be used as targeting moiety. Antitumor activity of the conjugated was evaluated in vitro using HeLa cells and the biotin conjugates presented much better effects than those without biotin. DOX release from the dendrimer was $\mathrm{pH}$-dependent, showing that the cleavage is potentially faster in slightly acidic environment. ${ }^{28}$ 

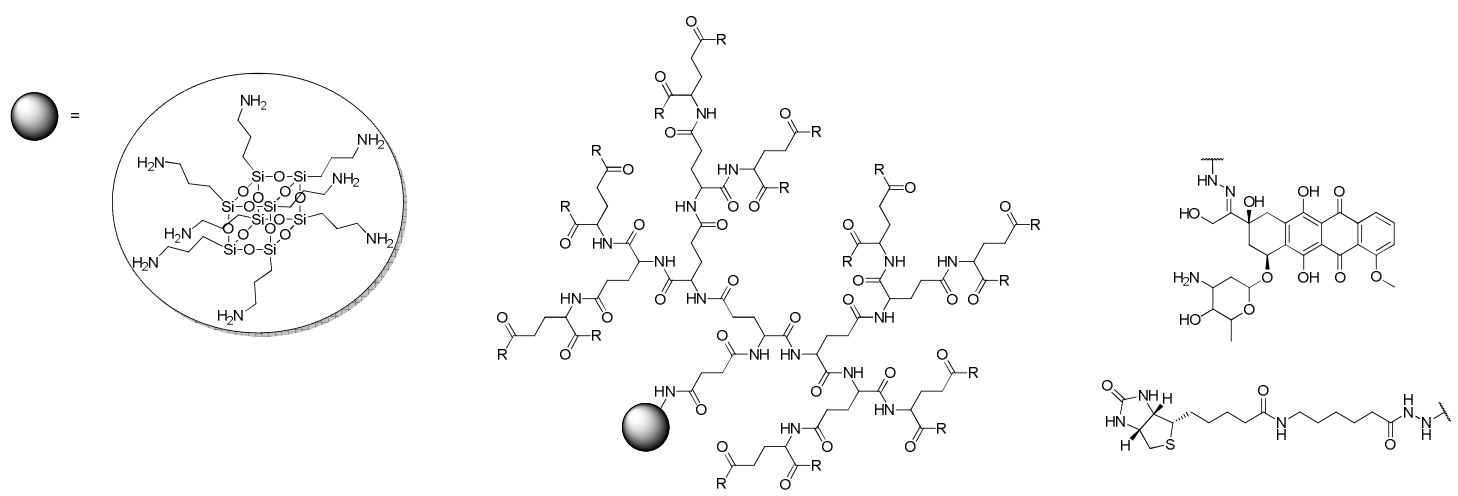

Figure 4 - Poly(L-glutamic acid) dendrimer with octa(3-aminopropyl) silsesquioxane (OAS) core conjugated with DOX in their peripheral groups through $\mathrm{pH}$-sensitive hydrazone bonds and biotin as a specific tumor cells targeting moiety.

A supramolecular self-assembly capsid-like nanocarriers (CLNs) were developed based on dendrimer systems for drug internalization, site-specific delivery, pH-responsiveness. This was bioinspired and synthetized to mimic viral capsids, able to encapsulate antitumor drug into hydrophobic cavity by non-covalent interaction and $\mathrm{pH}-\mathrm{dependent}$ disassembly for drug releasing. CLNs are constituted of poly(L-lysine) dendrimer with octa(3-aminopropyl) silsesquioxane core and connected by non-covalent bond to glutamic acid coupled poly(L-leucine) (Figure 5) and used for DOX delivery (D-CLN). The in vitro release profiles of D-CNL showed the conjugate had higher stability in $\mathrm{pH} 7.4$, while acidic condition $(\mathrm{pH}$ 6.2) used to mimic tumor intracellular environment, remarkably accelerated the drug release in the cytoplasm. This DOXdelivery system showed efficient tumor suppression, when assayed in BALB/c mice bearing 4T1 tumor, decreasing DOX-induced cardiotoxicity. ${ }^{19}$ 


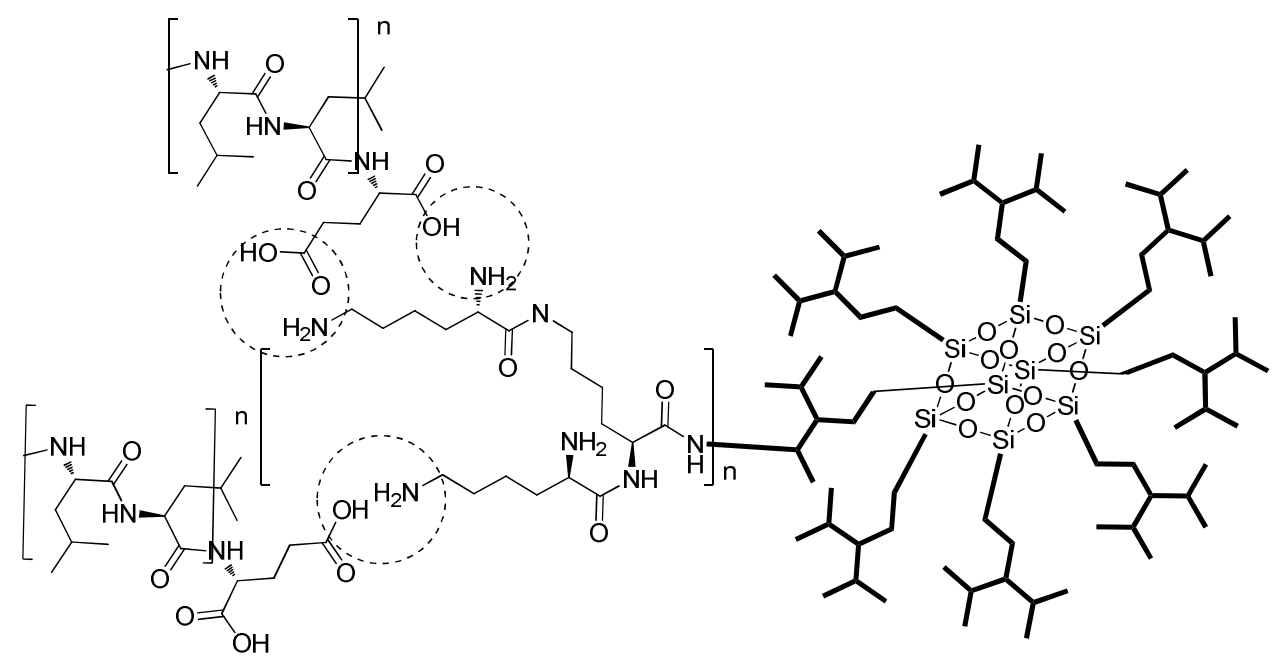

Figure 5 - Poly(L-lysine) dendrimer with octa(3-aminopropyl) silsesquioxane core and connected to non-covalent bond to glutamic acid coupled poly(L-leucine).

$\mathrm{Xu}$ and coworkers $^{39}$ (2011) developed a novel micellar system with a polypeptide dendrimer using a convergent-divergent approach. This system is composed of poly(glutamic acid) dendrimer of third generation, linked to poly(Lphenylalanine) polymer transformed into a core-shell structure, loaded with DOX. The polypeptide dendrimer block copolymer has the ability of self-assembly forming micelles and sustaining drug release. It exhibited efficient antitumor activity, inhibiting the proliferation of HepG2 liver cancer cells in in vitro incubation.

Metastatic cells degrade the collagen, which makes up the extracellular matrix. Based on this effect, PAMAM generation 4 was linked with type I and type IV collagens via glutamate ${ }^{40}$ and transformed into a peptide gel, attached to DOX by hydrazone bond, degradable at low $\mathrm{pH}$. This DOX-conjugated dendrimer/collagen hybrid gel systems were useful for metastasis-associated drug delivery systems. The prodrug obtained was evaluated in two cancer cell lines, MCF-7 and MDA-MB-231. A higher cytotoxic effect was observed in highly invasive MDA-MB-231. There was a greater cytotoxic to highly invasive MDA-MB-231 cell, compared with less invasive MCF-7 cells.

Other compounds, such as platinum derivatives, have been used for a long time to achieve antineoplastic effect. ${ }^{41}$ Despite the fact that oxaliplatin is a new generation drug to treat cancer, there are limitations to its use, such as neurotoxicity and inactivation due to its coordination with plasma proteins. ${ }^{42}$ Among the different approaches, the $\mathrm{pH}$-responsive bond has also been used to link the drug to the carrier. 
Previous studies focused on the development of new drug carriers to platinum group to increase the concentration of the metal in the tumor tissue. The use of linear copolymers demonstrated good $\mathrm{pH}$ stability in physiological conditions and increased blood residence time. Peptide dendrimers with less than $40 \mathrm{kDa}$ showed good biodegradability in addition to an increase in the blood residence time, displaying the ability to link to other ligands, by means of terminal groups, such as drugs or other modified molecules, as PEG.43

Conjugating PEGylated peptide dendrimer to diaminocyclohexyl-platinum (II), (DACHPt), is a strategy for drug delivery that could be used to treat ovarian cancer, as it enhances the concentration of platinum in the tumor tissue, decreasing toxic effects. Its use in cancer therapy has been evaluated and the conjugate had higher tumor activity in vivo than oxaliplatin. Moreover, it decreases toxic effects to normal tissues and the $\mathrm{pH}-$ sensitive bond is fast cleaved in acidic conditions. ${ }^{43}$

Based on the use of CPP-dendrimer derived (CPPD) for increasing the internalization of drugs, ${ }^{44,45}$ Zhao et al. ${ }^{44}$ (2014) investigated the cell penetrable lysine dendrimers conjugate with 5-fluororacil (G1-G3). The authors observed some advantages such as stability, low toxicity to normal cells, although moderate activity in tumors was demonstrated. They synthesized lysine dendrimer by convergent synthesis and evaluated cell penetration by labeling the conjugate with fluorescein isothiocyanate (FITC). Drug release, being stable in saline solution, occurred smoother and more sustainable with dendrimer G2 than with G1 and G3. Moreover, the normal cell uptake was lower than that in tumor cells, reducing the cytotoxicity and retaining a moderate anti-cancer activity.

Eggiman et al. $^{45}$ (2014) also detected an efficient cell internalization using CPPDs. They synthesized some peptide dendrimers and evaluated them with suitable techniques, showing moderate toxicity and stability in human serum after 12 hours, when compared with CPPs. Clathrin-mediated endocytosis displayed an important role in this process, as internalization was energy-dependent. Uptake was affected when the temperature decreased from $37{ }^{\circ} \mathrm{C}$ to $4{ }^{\circ} \mathrm{C}$, leading to membrane translocation. The drug used in the conjugate was paclitaxel, employed mainly for metastatic ovarium cancer. It is an insoluble drug, but its conjugate with CPPD showed to be soluble in water and stable all over the experiments. ${ }^{45}$

Bone cancer, although not common, has been a challenge to the chemotherapy. Some peptide-dendrimer systems have been studied looking for better drug delivery to 
the bones. Therefore, a peptide dendrimer was designed and synthetized for the treatment of bone cancer, consisting of two different dendrons: one is RGD (ariginineglycine-aspartic tripeptide) dimer and the other is 5-flouracil dimer that compose the first-generation Janus-type dendrimer. It is worth noting that Janus-type dendrimer is comprised of two dendrimeric wedges, having two different terminal functionalities. ${ }^{46}$ RDG has been reported in the literature as an interaction mediator between some integrins and drug delivery systems. Moreover, the $\alpha v \beta 3$ integrins are overexpressed in bone metastatic cells and osteoclasts, and, therefore, RDG can be used as targeting moiety to drug carrier. The in vitro assays demonstrated that the conjugate can bind to hydroxyapatite, which is the major inorganic bone component, and a potential sustained-release may reduce the side-effects. ${ }^{47}$

Although naproxen important adverse effects, it has the ability of inhibiting the development of bladder cancer. Moreover, it activates the caspase pathway, which induces apoptosis in breast cancer cells. In in vitro test, it can suppress cell migration and inhibit the synthesis of prostaglandin E-2 (PGE-2). Curcumin, a natural product extracted from the herb Curcuma longa, has therapeutic effects involving antiinflammatory, antioxidant and antitumor activities, especially against osteosarcoma, breast and colon cancer. When curcumin is combined with a naproxen prodrug, one can expect synergistic antitumor effects. Peptide dendrimers with good capacity to deliver drugs were earlier designed and synthesized, and those with glutamic or aspartic acids showed high selectivity to bone tissue. With these data, naproxen modified dendrimer with oligo-aspartic acid to encapsulate curcumin into micelles was developed to treat osteosarcoma. After the encapsulation, curcumin disperse with naproxen-dendrimer can rapidly release from the micelles during in vitro assays. Curcumin micelle showed greater cytotoxicity to cancer cells than free curcumin and naproxen-dendrimer alone, inducing more apoptosis through a mitochondrial pathway. Micelle encapsulation allowed the absorption of curcumin by the cancer cells more selectively. ${ }^{48}$

A more complex peptide dendrimer was designed by Ma et al., in $2017 .{ }^{49}$ It is known that arginine-lysine-glycine peptide (RGD) have high affinity to integrin $\alpha v \beta 3$ (it is overexpressed in tumors cells) being a promising target. ${ }^{50}$ Based on this tripeptide and on the TAT-peptide (GRKKRRQRRRPQ), which is derived from the transcription transactivator (TAT) of human immunodeficiency virus, a CPP, the authors tried to enhance drug uptake in tumors cells, conjugating it to RGD and PAMAM dendrimer. The problem of CPP nonspecific internalization, was overcome by PEG addition. The 
authors synthesized the conjugate RGB-TAT-PEG-PAMAM with methotrexate (MTX), a known anti-cancer drug, and, together with a probe (FITC), it was encapsulated. Flow cytometry showed that RGD-TAT-PAMAM-PEG-FITC (RTPPF) uptake was higher than RGD-PAMAM-PEG-FITC (RPPF). in $\alpha v \beta 3$ overexpressing HepG2. In confocal microscopy the conjugates were accumulated in HepG2 cells and co-localized at cell nucleus. The difference between them was the fluorescence intensity, which was stronger in RTPPF than in RPPF. Despite the fact that both could suffer endocytosis, RTPPF had better internalization. MTX complexes were more slowly eliminated than MTX and had 2-fold higher maximum tolerated regimen values.

\section{Delivery systems for other drugs}

The carbohydrates interaction with specific cell receptors, such as macrophages and liver cells, is used as a selective strategy, as mentioned before.

The poly(L-lysine) dendrimer four generation with PEG as core, and conjugated with D-galactose, is one example of this approach. This dendrimer interacts with the asialo-glycoprotein receptor in the liver, which recognizes galactose. The glycopeptide dendrimer was used to encapsulate chloroquine phosphate (CQ). The results obtained in vitro and in vivo, compared with the galactose-uncoated peptide dendrimer, showed that the latter allowed a slower drug release rate than the former, besides half-reducing the drug hemolysis rate. There was also a 5-time reduction in the macrophage uptake of this dendrimer. Therefore, data evidenced that coated peptide dendrimers were the most effective towards antimalarial target to the liver. ${ }^{51}$

Other glycoconjugated peptide dendrimers were designed by Bhadra et al. (2006). ${ }^{52}$ They synthesized coated PEG-lysine dendrimers (PEG-Lys-CSA) and uncoated (PEG-Lys) with chondroitin sulfate A, encapsulating chloroquine phosphate (CQ) in both carriers, suggesting that dendrimer matrix prolonged the drug releasing time. This could be associated with the increase in PEG molecular weight and in dendrimer generations. However, in CSA-coated dendrimers, the increased viscosity and lack of free periphery groups led to a compact structure. The latter was less hemolytic than CQ and PEG-Lys-CQ, which might have masked free groups and charges of drug molecules. In the coated dendrimer, the uptake by macrophages was less than that observed for CQ itself. In addition, that compound was nearly 50\% less cytotoxic, having a safe increasing blood circulation time. Although both dendrimers had some of their pharmacokinetics parameters increased, PEG-Lys-CAS-CQ retarded 
the drug release in more than 12 hours. Due to the dendrimer circulation increase, CQ was more available in blood than in liver and spleen with PEG-Lys-CAS-CQ.

Musculoskeletal diseases, as osteoarthritis and osteoporosis, represent an important public health problem, arousing great interest in research and development of drug-delivery systems that can efficiently transport the drug to the bone. These kinds of systems are especially important once we know that the bones, unlike other parts of the body, have a layer of hydroxyapatite, and some drugs, such as tetracyclines and bisphosphonates, display a high affinity to this substance. Therefore, these substances could work as targeting groups to bone. ${ }^{53}$

Giarolla and colleagues have designed peptide dendrimers Lys-Arg-based aiming at obtaining cleavage by cruzain, which is a promising target in the planning of new antichagasic compounds. Peptide dendrimers were designed with molecules potentially active in Chagas' Disease, having their main objective to achieve the release of the active compound directed in the protozoan. ${ }^{54}$

\section{Gene delivery}

Gene therapy is one of the most studied treatment for human diseases such as cancer and diabetes, usually, resulting in the knockdown dysfunctional genes of or in the increase of genes expression. One of challenges concerning this treatment is the gene products delivery to the site of action. ${ }^{55,56}$

Despite being the greatest technique used for gene delivery, viral vectors still have some problems such as mutagenicity and immune reactions. Several nanocarriers, from liposomes to dendrimers, present the key characteristics for being an efficient gene delivery, which can prolong blood circulation time, almost without immune reaction, high cellular uptake and early endosomal scape. ${ }^{55}$

Unlike the typical dendrimers, the peptide ones have some unique well-defined properties, low polydispersity, high surface charges density, and highly adaptable chemical surface. However, peptide dendrimers have biological barriers and cytotoxicity to overcome. $^{57}$

The cationic polymeric carrier succeeded much better in the negatively charged DNA condensation. Among the cationic polymers, polyethylenimine (PEI) has shown to be the best, mostly because of the high cellular uptake and endosomal scape. However, PEI transporter is so positively charged, that can cause membrane disruption leading to cell death. PAMAM dendrimers have the ideal structure to be an efficient 
gene delivery. Thus, Choi et al. $(2004)^{56}$ created an arginine functionalized PAMAM dendrimer, testing and comparing it with regular PAMAM, PEI and lysine functionalized PAMAM. The results showed PAMAM-Arg as promising to gene delivery, since it enhanced the expression of HepG2 and Neuro2A cell lines in primary rat vascular smooth muscles, showing to be better than the regular PAMAM and PAMAM-Lys. Although PAMAM-Arg presented less potential as gene delivery than PEI, it has much less cytotoxicity due to fewer positive moieties. ${ }^{56,58}$

Another interesting study developed by Zarebkohan et al. (2015) about dendrimers was related to gene delivery to the brain $^{59}$ The authors used 4 th generation PAMAM dendrimer functionalized with SRL (serine-arginine-leucine), which is relevant as gene delivery, aming at crossing the blood-brain barrier (BBB). Moreover, comparing with PAMAM-arg and PAMAM-DAN particle, the results showed better uptake to the brain.

PAMAM derivative compounds were also built with phenylalanine and plasmid DNA in their periphery as agents for gene delivery. One of the dendrimers showed to be an efficient transfection agent, better than the commercially available transfection reagent PEI (polyethylenimine) and other derivatives from the series. ${ }^{60}$

Many authors agree that the biological barrier and the cytotoxicity are problems related to high generation peptide dendrimers. Luo et al. $(2012)^{61}$ synthesized a 5 th and 6th generation-peptide dendrimers, functionalized with arginine, and studied their success in gene delivery, as well as their cytotoxicity. The results displayed the 5th generation dendrimer as being the best gene delivery system. Additionally, this compound presented much higher cell viability, when compared with 6th generation. The authors advanced a hypothesis related to the density of positively charged of primary amines in their surface. This high density of positive charges can cause an interaction between the dendrimer and the negatively charged cell membrane, producing its disruption. ${ }^{51}$

Considering dendrimers having low biodegradability and biocompatibility, Cai et al. $(2016)^{62}$ developed a fluorinated peptide dendrimer, evaluating its potential to be an effective gene delivery. The fluorination of the molecule resulted in less protein interaction, increase in cellular uptake, endosome scape and cytoplasm trafficking, showing an increased biocompatibility and biodegradability.

\section{Other applications}


Peptide dendrimers can display activity per se, in diagnosis, to stimulate immune response, as vaccines.

\section{Diagnostic, biosensor and nanodiagnostics}

MAP (Multiple Antigen Peptides) dendrimers presented better specificity to some antibodies than linear peptides in ELISA tests. Moreover, it has been used in many immunoassays to identify antigen, such as HIV, malaria, BTV (blue tongue virus), infectious bronchitis and other pathogens ${ }^{63}$. MAP dendrimers may have more than one different epitopes in the serodiagnosis of hepatitis $\mathrm{C}$ virus. ${ }^{64,65}$ Additionally, those dendrimers, acting as biosensors, could be used as probes to detect bioorganic compounds. $^{64}$

\section{Immune response and vaccines}

Peptide dendrimers can be used to detect antibodies or immune responses with good sensitivity, for instance, that from PPR (Peste des petits ruminant virus) virus. ${ }^{64,65}$

Roy and coworkers published the first paper regarding glycopeptide dendrimers, in $1993 .{ }^{62}$ The researchers reported the synthesis of a peptide dendrimer composed of lysine and sialic acid as coating antigen, which showed excellent antigenic inhibitory capacity. Many other architectures of glycopeptide dendrimers, potentially used as vaccines, were described in an excellent review published by Roy, Shiao and Rittenhouse-Olson (2013). ${ }^{67}$

MAP, having better protection profile, are used to enhance the immune response and are more chemically stable depending on their constitution, which can be homotropic (multimer with one epitope) or heterotropic (multimer with different epitopes). Some studies have shown the vaccine application of MAP dendrimers in influenza type $\mathrm{A}$, hepatitis $\mathrm{A}$ and $\mathrm{C}$ virus, respiratory syncital virus, and classical swine fever. However, dendrimer vaccine faces some limitations such as neutralization, in many cases, delivery and biostability. ${ }^{64}$

\section{Antimicrobial and antiviral actions}

Some peptide dendrimers are described in the literature for their efficient antimicrobial and antiviral properties. As antiviral, MAP dendrimers generally act in two ways, either binding to virus receptors on cell surface or mimicking cell receptor. ${ }^{45}$ Some studies, which used a dendrimer heparin-sulfate biding peptide, led to the 
inhibition of Human papillomavirus, Human cytomegalovirus and Herpes simplex virus types 1 and $2 .^{64}$

A common use to the treatment of HSV (herpes simplex virus) infections are synthetic nucleoside analogs targeting viral DNA polymerase. However, long treatments may fail because of virus resistance. Trying to solve this limitation, Tarallo and colleagues ${ }^{68}$ (2013) investigated poly(amide)-based dendrimers as antiviral agents. Some dendrimers received a gH625 peptide (membranotropic peptide sequence), which interact with the membrane bilayer and possess some antiviral activity. Tested in African green monkey kidney cells (Vero), these dendrimers together with propidium iodide to distinguish apoptosis from necrosis, showed that the inhibition of infectivity may be due to the formation of inactive aggregates. Moreover, gH625 peptide coupled to the dendrimer was effective to prevent viral entry and viral infectivity. HSV virus presented a consistent reduction in replication and more than $80 \%$ of inhibition. On the other hand, dendrimer without any peptide produced only $35 \%$ of inhibition, suggesting this structure had some antiviral activity. It is worth considering that low toxicity, once approximately $90 \%$ of cells treated with both dendrimers survived. ${ }^{64}$

There are peptides, known as antimicrobial peptides (AMP), which have antimicrobial activity by themselves and are produced by system defense of multicellular organisms. Generally, the molecule contains residues of arginine and lysine, 30\% hydrophobic side chains and amphipathic conformation leading to a membrane disruption. ${ }^{65}$ Based on this AMP, Stach and coworkers ${ }^{69}$ (2014) designed and studied antimicrobial peptide dendrimers (AMPD), which are constituted of up to 40 residues, with multiple short mono-, di-, or tripeptide branches. These dendrimers were more resistant to proteolysis, because of their molten globule-like conformation, which led to 200-fold less hemolysis. To test the antimicrobial activity, they used three bacterium species, namely P. aeruginosa, E. coli and B. subtilis. The results suggested that the $3^{\text {rd }}$ generation dendrimer is more active than the lower ones. In addition, it seemed that the amino acid sequence displayed a role in the activity, since changes in the composition decreased the activity and increased the hemolytic resistance. It is worth mentioning that the AMPD showed to be active in resistant strains and also stable, presenting strong activity in human serum. Using the same approach, Pires and colleagues $^{70}$ (2015) developed a novel AMPD, G3KL (Figure 6) and evaluated in vitro activity against $32 \mathrm{~A}$. baumannii and $35 \mathrm{P}$. aeruginosa isolates collected in distinct countries during different periods. MIC was lower or similar to standard antibiotics 
except for colistin and polymyxin B, which showed to be more active. G3KL is a promising active antibacterial molecule, which can potentially be applied clinically in the future, once it showed results for both species with multidrug-resistant strains and extensively drug-resistant $A$. baumannii and $P$. aeruginosa.

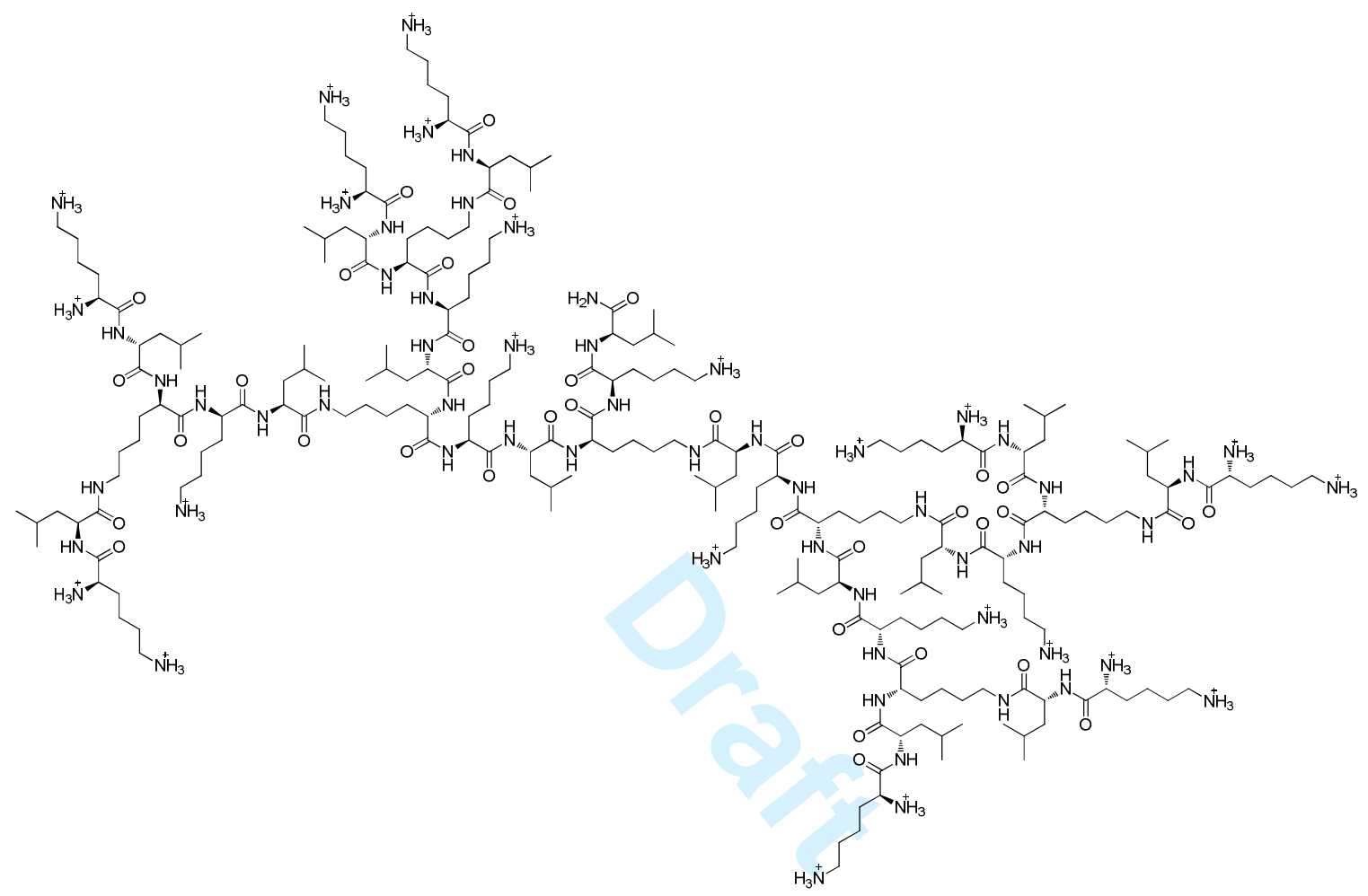

Figure 6 - Novel antimicrobial peptide dendrimers (AMPD), G3KL.

The human pathogen Pseudomonas aeruginosa infects immune-compromised and cystic fibrosis-patients, causing lethal airway infections. This bacterium can form a lectin biofilm, LecA and LecB, which are barriers to antibiotics, since they inhibit biofilm formation by multivalence and dispersion. Moreover, it is important to develop inhibitors for them. ${ }^{71}$ Based on this, Reymond, Bergmann and Darbre ${ }^{71}$ (2013) synthesized two kinds of glycopeptide dendrimers: fucosylated peptide dendrimer (FD2) and galactosylated dendrimer (GalAG2 and GalBG2), which improved lectine targeting in order to increase biofilm inhibition. The latter interacted with lectin LecA.

The same group ${ }^{72}$ designed heteroglycopeptide dendrimers with LecA and LecB, incorporating cationic residues in the dendrimers. The additional positive charges showed to increase biofilm inhibition and bactericide effect, similar to other polycationic dendrimers. Noteworthy, biofilm inhibition and dispersion could be 
obtained by co-application of dendrimer FD2 and tobramycin, suggesting that this combination may be used in synergy to restore antibiotic effects.

Lind and colleagues ${ }^{73}$ (2013) synthesized a novel amphiphilic peptide dendrimer (BALY) with potential application against multi-resistant bacteria (Figure 7) The activity was evaluated in $S$. aureus ATCC 25923, methicillin resistant $S$. aureus ATCC 43300 (MRSA), E. coli 25922 and P. aeruginosa. The results showed MIC $\sim 1 \mu \mathrm{M}$ against Gram-positive $S$. aureus with cell lysis and 10-fold selectivity over Gramnegative strains. The evaluation in situ using combination of quartz crystal microbalance and atomic force microscopy imaging showed that those dendrimers were able to destroy the membrane integrity through different mechanisms depending on the lipid phase and morphology.

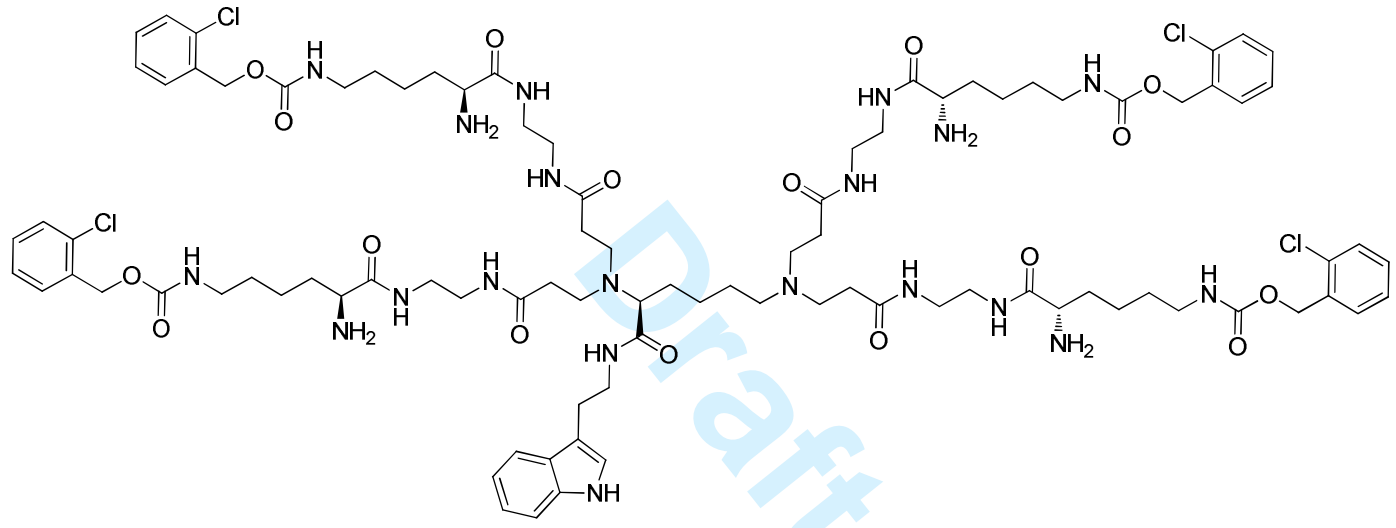

Figure 7 - Novel amphiphilic peptide dendrimer (BALY) with potential application against multi-resistant bacteria.

Enzymes

Peptide dendrimers can be applied as artificial enzymes, as esterase, for instance. These dendrimers were designed employing proteinogenic R-amino acids with branches containing diamino acids, with molecular weights ranging from 3 to $5 \mathrm{kDa}$. They were synthesized by solid-phase methodology, purified and dimerized by disulfide bond using cysteine residues. Combinations of aspartate, histidine, and serine, amino acids usually present in esterases and lipases, were coupled. ${ }^{74}$

\section{CONCLUDING REMARKS}

The use of peptide dendrimers for their potential applications must be encouraged. Although peptide synthesizes well-developed, dendrimers, on the other hand, also deserve growing interest. The association of the former and the latter must be 
stimulated since it can generate better alternatives in terms of efficacy, synergism and selectivity of action. In this review, the main application of peptide dendrimers was on drug delivery systems, especially in cancer, which continues to be a huge challenge for the therapeutics. Nanocarriers, derived from peptide dendrimers, can also be used as gene delivery systems, but this area deserves more improvement, taking into account its complexity. Exploring the activity per se of these compounds is another interesting possibility of findings, especially, antinfectious agents. Compounds to bypass the problem of resistance are urgently needed. In all those areas the versatility of the peptide dendrimers, especially considering the possibility of using $\mathrm{pH}$-sensitive linkage, self-assembly forming micelles, and the conjugation with different molecular architectures, is a quality that has to be better scrutinized toward innovation.

\section{Acknowledgments}

The authors are grateful to Fundação de Amparo à Pesquisa do Estado de São Paulo (FAPESP) for Shikay JM scholarship (2016/11555-1) and financial support for Giarolla J (2015/19438-1). We also thank Conselho Nacional de Desenvolvimento Científico e Tecnológico for Silva RS, Silva JV for scholarships and for Ferreira EI research fellowship, and Coordenação de Aperfeiçoamento de Pessoal de Nível Superior (CAPES) for Gonzaga RV scholarship.

\section{Conflicts of Interest}

The authors confirm no conflict of interest.

\section{REFERENCES}

(1) Sadler, K.; Tam, J. P. Rev. Mol. Biotechnol. 2002, 90 (3-4), 195.

(2) Sebestik, J.; Niederhafner, P.; Jezek, J. Amino Acids. 2011, 40 (2), 301.

(3) Wan, J.; Alewood, P. F. Angew. Chemie - Int. Ed. 2016, 55 (17), 5124.

(4) Schellinger, J. G.; Danan-Leon, L. M.; Hoch, J. A.; Kassa, A.; Srivastava, I.; Davis, D.; Gervay-Hague, J. J. Am. Chem. Soc. 2011, 133 (10), 3230.

(5) Skwarczynski, M.; Zaman, M.; Urbani, C. N.; Lin, I. C.; Jia, Z.; Batzloff, M. R.; Good, M. F.; Monteiro, M. J.; Toth, I. Angew. Chemie - Int. Ed. 2010, 49 (33), 5742. 
(6) Crespo, L.; Sanclimens, G.; Pons, M.; Giralt, E.; Royo, M.; Albericio, F. Chem. Rev. 2005, 105 (5), 1663.

(7) Machado, A.; Liria, C. W.; Proti, P. B.; Remuzgo, C.; Miranda, M. T. M. Quim. Nova. 2004, 27 (5), 781.

(8) Tam, J. P. Proc. Natl. Acad. Sci. U. S. A. 1988, 85 (15), 5409.

(9) Darbre, T.; Reymond, J. L. Acc. Chem. Res. 2006, 39 (12), 925.

(10) Niederhafner, P.; Sebestik, J.; Jezek, J. J Pept Sci. 2005, 11 (12), 757.

(11) Niederhafner, P.; Sebestik, J.; Jezek, J. J Pept Sci. 2008a, 14 (2), 43.

(12) Niederhafner, P.; Sebestik, J.; Jezek, J. J Pept Sci. 2008b, 14 (1), 44.

(13) Niederhafner, P.; Reinis, M.; Sebestik, J.; Jezek, J. J Pept Sci. 2008c, 14 (5), 56.

(14) Pérez, Y. A.; Urista, C. M.; Martínez, J. I.; Nava, M. D. C. D.; Rodríguez, F. A. R. Polymers. 2016, 8 (6), 214.

(15) Santos, S. S.; Ferreira, E. I.; Giarolla, J. Molecules. 2016, 21 (6), 686.

(16) Ahmed, S.; Vepuri, S. B.; Kalhapure, R. S.; Govender, T. Biomaterials Sci. 2016, 4 (7), 1032.

(17) Pomares, L. M. J. Leuk. Biol. 2012, 92 (6), 1177.

(18) Hsu, H. -J.; Bugno, J.; Lee, S. -ri Hong, S. WIREs Nanomed. Nanotechnol. 2017, 9 (1), 1 .

(19) Li, Y.; Lai, Y.; Xu, X.; Zhang, X.; Wu, Y.; Hu, C.; Gu, Z. Nanomed. Nanotechnol. Biol. Med. 2016, 12 (2), 355.

(20) Shadrack, D. M.; Mubofu, E. B.; Nyandoro, S. S. Int. J. Mol. Sci. 2015, 16 (11), 26363.

(21) Zhao, Y.; Zeng, Q.; Wu, F.; Li, J.; Pan, Z.; Shen, P.; Yang, L.; Xu, T.; Cai, L.; Guo, L.; Yamamura, S.; Gotoh, H.; Sakamoto, Y.; Momose, Y.; Ullrich, C. A.; College, H.; Nunes, C.; Brezesinski, G.; Lima, J. L. F. C.; Reis, S.; Lucio, M.; Koumaki, E.; Mamais, D.; Noutsopoulos, C.; Nika, M. C.; Bletsou, A. A.; Thomaidis, N. S.; Eftaxias, A.; Stratogianni, G.; Cuquerella, M. C.; Andreu, I.; Soldevila, S.; Bosca, F.; Cosa, G.; Lukeman, M.; Scaiano, J. C.; Carvalho, T. C.; Escotet, M. L.; Lin, J.; Sprockel, O. L.; Bani-Yaseen, A. D. Phys. Chem. Chem. Phys. 2016, 42 (6), 21322.

(22) Verma, R. P.; Shandilya, A.; Haridas, V. Tetrahedron. 2015, 71 (46), 8758.

(23) Mutalik, S.; Parekh, H. S.; Anissimov, Y. G.; Grice, J. E.; Roberts, M. S. Skin Pharmacol. Physiol. 2013, 26 (3), 127. 
(24) Yan, C.; Gu, J.; Hou, D.; Jing, H.; Wang, J.; Guo, Y.; Katsumi, H.; Sakane, T.; Yamamoto, A. Drug Dev. Ind. Pharm. 2015, 41 (4), 617.

(25) Jiang, Y.; Lv, L.; Shi, H.; Hua, Y.; Lv, W.; Wang, X.; Xin, H.; Xu, Q. Colloids Surfaces B Biointerfaces. 2016, 147, 242.

(26) Zugazagoitia, J.; Guedes, C.; Ponce, S.; Ferrer, I.; Molina-Pinelo, S; Paz-Ares, L. Clin. Ther. 2016, 38 (7), 1551.

(27) Yang, H. Nanomed. Nanotechnol. Biol. Med. 2016, 12 (2), 309.

(28) Yuan, H.; Luo, K.; Lai, Y.; Pu, Y.; He, B.; Wang, G.; Wu, Y.; Gu, Z. Mol. Pharm. 2010, 7 (4), 953.

(29) Shah, N. D.; Parekh, H. S.; Steptoe, R. J. Pharm. Res. 2014, 31 (11), 3150.

(30) Oledzka, E.; Sliwerska, P.; Sobczak, M.; Kraska, B.; Kamysz, W.; Nalecz-Jawecki, G.; Kolodziejski, W. Macromol. Chem. Phys. 2015, 216 (12), 1365.

(31) Gali-Muhtasib, H.; Hmadi, R.; Kareh, M.; Tohme, R. Darwiche, N. Apoptosis. 2015, 20 (12), 1531.

(32) Tacar, O.; Sriamornsak, P.; Dass, C. R. J. Pharm. Pharmacol. 2013, 65 (2), 157.

(33) She, W.; Li, N.; Luo, K.; Guo, C.; Wang, G.; Geng, Y.; Gu, Z. Biomaterials. 2013, $34(9), 2252$

(34) Li, N.; Li, N.; Yi, Q.; Luo, K.; Guo, C.; Pan, D.; Gu, Z. Biomaterials. 2014, 35 (35), 9529.

(35) Lee, S. J.; Jeong, Y. Il; Park, H. K.; Kang, D. H.; Oh, J. S.; Lee, S. G.; Lee, H. C. Int. J. Nanomedicine. 2015, 10 (1), 5489.

(36) Li, N.; Guo, C.; Duan, Z.; Yu, L.; Luo, K.; Lu, J.; Gu, Z. J. Mater. Chem. B. 2016, 4 (21), 3760 .

(37) LexInnova. Nanoparticles smart drug delivery system for cancer. Technology landscape. www.lexinnova.com. Accessed: February, 2016.

(38) Liu, D.; Yang, F.; Xiong, F.; Gu, N. Theranostic. 2016, 6 (9), 1306.

(39) Xu, X.; Li, C.; Li, H.; Liu, R.; Jiang, C.; Wu, Y.; He, B.; Gu, Z. Sci. China Chem. 2011, 54 (2), 326.

(40) Kojima, C.; Suehiro, T.; Watanabe, K.; Ogawa, M.; Fukuhara, A.; Nishisaka, E.; Harada, A.; Kono, K.; Inui, T.; Magata, Y. Acta Biomater. 2013, 9 (3), 5673.

(41) Harper, B. W.; Krause-Heuer, A. M.; Grant, M. P.; Manohar, M.; GarbutcheonSingh, K. B.; Aldrich-Wright, J. R. Chem. Eur. J. 2010, 16 (24), 7064.

(42) Carozzi, V. A.; Canta, A.; Chiorazzi, A. Neurosci. Lett. 2015, 596, 90. 
(43) Pan, D.; She, W.; Guo, C.; Luo, K.; Yi, Q.; Gu, Z. Biomaterials. 2014, 35 (38), 10080 .

(44) Zhao, J.; Zhou, R.; Fu, X.; Ren, W.; Ma, L.; Li, R.; Zhao, Y.; Guo, L. Arch. Pharm. (Weinheim). 2014, 347 (7), 469.

(45) Eggimann, G. A.; Blattes, E.; Buschor, S.; Biswas, R.; Kammer, S. M.; Darbre, T.; Reymond, J.-L. Chem. Commun. (Camb). 2014, 50 (55), 7254.

(46) Caminade, AM.; Majoral, J-P. Molecules. 2016, 21 (4), 538.

(47) Jiang, B.; Zhao, J.; Li, Y.; He, D.; Pan, J.; Cao, J.; Guo, L. Lett. Org. Chem. 2013, $10(8), 594$.

(48) Zhao, Y.; Zeng, Q.; Wu, F.; Li, J.; Pan, Z.; Shen, P.; Yang, L.; Xu, T.; Cai, L.; Guo, L. RSC $A d v$. 2016, 6 (65), 60327.

(49) Ma, P.; Yu, H.; Zhang, X.; Mu, H.; Chu, Y.; Ni, L.; Xing, P.; Wang, Y.; Sun, K. Pharm. Res. 2017, 34 (1), 121.

(50) Zhu, S.; Qian, L.; Hong, M.; Zhang, L.; Pei, Y.; Jiang, Y. Adv. Mater. 2011, 23 (12), 84 .

(51) Agrawal, P.; Gupta, U.; Jain, N. K. Biomaterials. 2007, 28 (22), 3349.

(52) Bhadra, D.; Bhadra, S.; Jain, N. K. Pharm. Res. 2006, 23 (3), 623.

(53) Ouyang, L.; Huang, W.; He, G.; Guo, L. Lett. Org. Chem. 2009, 6 (4), 272.

(54) Giarolla, J.; Silva, J.V.; Shikay, J.M.; Machini, M.T. Non published data.

(55) Thuy, L. T.; Mallick, S.; Choi, J. S. Int. J. Pharm. 2015, 492 (1-2), 233.

(56) Choi, J. S.; Nam, K.; Park, J. Y.; Kim, J. Bin; Lee, J. K.; Park, J. S. J. Control. Release 2004, 99 (33), 445.

(57) Dufès, C.; Uchegbu, I. F.; Schätzlein, A. G. Adv. Drug Deliv. Rev. 2005, 57 (15), 2177.

(58) Liu, Z.; Zhang, Z.; Zhou, C.; Jiao, Y. Prog. Polym. Sci. 2010, 35 (9), 1144.

(59) Zarebkohan, A.; Najafi, F.; Moghimi, H. R.; Hemmati, M.; Deevband, M. R.; Kazemi, B. Eur. J. Pharm. Sci. 2015, 78, 19.

(60) Wang, X.; He, Y.; Wu, J.; Gao, C.; Xu, Y. Biomacromolecules. 2010, 11 (1), 245.

(61) Luo, K.; Li, C.; Li, L.; She, W.; Wang, G.; Gu, Z. Biomaterials. 2012, 33 (19), 4917.

(62) Cai, X.; Jin, R.; Wang, J.; Yue, D.; Jiang, Q.; Wu, Y.; Gu, Z. ACS Appl. Mater. Interfaces. 2016, 8 (9), 5821.

(63) Tam, J. P.; Zavala, F. J. Immunol. Methods 1989, 124 (1), 53. 
(64) Joshi, V. G.; Dighe, V. D.; Thakuria, D.; Malik, Y. S.; Kumar, S. Indian J. Virol. 2013, 24 (3), 312.

(65) Dechamma, H. J.; Dighe, V.; Kumar, C. A.; Singh, R. P.; Jagadish, M.; Kumar, S. Vet. Microbiol. 2006, 118 (3-4), 201.

(66) Roy, R.; Zanini, D.; Meunier, S. J.; Romanowska, A. J. Chem. Soc. Chem. Commun. 1993, (24), 1869.

(67) Roy, R.; Shiao, T.C.; Rittenhouse-Olson, K. Braz. J. Pharm. Sci. 2013, 49 (SI), 85.

(68) Tarallo, R.; Carberry, T. P.; Falanga, A.; Vitiello, M.; Galdiero, S.; Galdiero, M.; Weck, M. Int. J. Nanomedicine 2013, 8, 521.

(69) Stach, M.; Siriwardena, T. N.; Köhler, T.; Van Delden, C.; Darbre, T.; Reymond, J. L. Angew. Chemie - Int. Ed. 2014, 53 (47), 12827.

(70) Pires, J.; Siriwardena, T.N.; Stach, M.; Tinghely, R.; Kasraian, S.; Luzzaro, F.; Leib, S.L.; Darbre, T.; Reymond, J.-L.; Endimiani, A. Antim. Agents Chemother. 2015, 59 (12), 7915.

(71) Reymond, J-L.; Bergmann, M.; Darbre, T. Chem. Soc. Rev. 2013, 42 (11), 4814.

(72) Michaud, G.; Visini, R.; Bergmann, M.; Salerno, G.; Bosco, R.; Gillon, E.; Richichi, B.; Nativi, C.; Imberty, A.; Stocker, A.; Darbre, T.; Reymond, J-L. Chem. Sci. 2016, 7 (1), 166.

(73) Lind, T. K.; Zieli, P.; Al, L. E. T. 2013, No. 1, 396.

(74) Esposito, A.; Delort, E.; Lagnoux, D.; Djojo, F.; Reymond, J. L. Angew. Chemie Int. Ed. 2003, 42 (12), 1381. 